\title{
Evidence for downregulation of the negative regulator SPRED2 in clinical prostate cancer
}

\author{
N Kachroo ${ }^{1,3}$, T Valencia ${ }^{1,3}$, A Y Warren ${ }^{2}$ and V J Gnanapragasam*,1 \\ ${ }^{1}$ Translational Prostate Cancer Group, Hutchison/MRC Research centre, University of Cambridge, Cambridge CB1 OXZ, UK and \\ ${ }^{2}$ Department of Pathology, Addenbrookes Hospital, Hills Road, Cambridge CB2 OXZ, UK
}

Background: SPRED1 and 2 are key negative regulators of MAPK signalling in mammalian cells. Here, we investigate the expression and functional role of SPREDs in prostate cancer.

Methods: A transcriptome bank of microdissected grade-specific primary cancers was constructed and interrogated for transcript expression of prostate cancer genes, known negative signalling regulators as well as SPRED1 and 2 . The effect of SPRED2 manipulation was tested in in vitro assays.

Results: In a panel of 5 benign glands and 15 tumours, we observed concomitant downregulation of the negative regulators SEF and DUSP1 in tumours with increasing Gleason grade. Profiling in the same cohorts revealed downregulation of SPRED2 mRNA in tumours compared with benign glands $(P<0.05)$. By contrast, SPRED1 expression remained unchanged. This observation was further validated in two additional separate cohorts of microdissected tumours (total of $n=10$ benign and $n=58$ tumours) with specific downregulation of SPRED2 particularly in higher grade tumours. In functional assays, SPRED2 overexpression reduced ERK phosphorylation and inhibited prostate cancer cell proliferation and migration in response to different growth factors and fullmedia stimulation $(P<0.001)$. Conversely, SPRED2 suppression by siRNA enhanced the mitogenic response to growth factors and full media $(P<0.001)$.

Conclusion: These data suggest first evidence that SPRED2 is downregulated in prostate cancer and warrants further investigation as a potential tumour-suppressor gene.

Enhanced intracellular signalling is an important mechanism contributing to cancer progression (Brognard et al, 2011). Intracellular signalling is normally subject to constitutive levels of regulation that modulate the level of intracellular signalling (Murphy et al, 2010). Negative regulators provide a feedback mechanism that controls the intensity and duration of exogenous stimulation and have been particularly implicated in modulation of the MAPK pathway. Key members of this group, including the MAPK phosphatases, RKIP1, SPROUTY and SEF, have been consistently reported to be downregulated in many malignancies, including prostate cancer (Murphy et al, 2010). The SPROUTYrelated enabled/vasodilator-stimulated phosphoprotein homology 1 domain-containing (SPRED) proteins (SPRED1 and 2) were first described by Wakioka et al (2001). SPREDs function by forming a complex with Raf and inhibiting activation of MEK (Wakioka et al,
2001; Bundschu et al, 2007). To date, there is very limited data on expression levels of SPRED1 and 2 in human cancers. SPREDs have only been investigated in hepatocellular carcinoma, with an observed downregulation of SPRED1 and 2 (Yoshida et al, 2006). Decreased SPRED levels were also associated with increased tumour invasion and metastasis (Yoshida et al, 2006; Ma et al, 2011). Given known patterns of loss of other signalling regulators in prostate cancer, we investigated whether expression of SPREDs was similarly altered.

\section{MATERIALS AND METHODS}

Tumour microdissection, RNA isolation and real-time PCR. Benign and malignant areas of distinct grades (Gleason grades 3, 4

*Correspondence: Dr VJ Gnanapragasam; E-mail: vjg29@cam.ac.uk
${ }^{3}$ These authors contributed equally to this work

Revised 14 September 2012; accepted 16 October 2012; published online 20 November 2012

(C) 2013 Cancer Research UK. All rights reserved 0007-0920/13 
or 5) from diagnostic prostate needle biopsies were laser microdissected, and RNA was extracted as previously described (ethics 09/H0308/42; Rogerson et al, 2008; Valencia et al, 2011). cDNA was synthesised (Transcriptor, Roche Diagnostics, Mannheim, Germany) and pre-amplified (Taqman PreAmp Mastermix, Applied Biosystems, Warrington, UK) according to the manufacturer's protocol. Real-time PCR conditions comprised of denaturation at $95^{\circ} \mathrm{C}$ for $10 \mathrm{~min}, 40$ cycles of $10 \mathrm{~s}$ at $95^{\circ} \mathrm{C}$ and $30 \mathrm{~s}$ at $60^{\circ} \mathrm{C}$. Gene expression was performed by relative quantification using GAPDH as the reference. Results are the mean of three separate triplicates.

Cell lines, transfection and functional assays. PC3M and LNCaP cells were maintained in RPMI (Gibco, Paisley, UK) alone or with $10 \%$ foetal bovine serum (basal and full medium (FM)). For androgen induction assays, LNCaP cells were grown in FM, then deprived of androgens (RPMI $+10 \%$ charcoal-stripped serum) for $24 \mathrm{~h}$ before treatment with ethanol (control) or $10 \mathrm{nM}$ of R1881. RNA was isolated using the RNeasy Mini Kit (Qiagen, Crawley, UK). In functional assays, cells were transfected (Lipofectamine $200)$ with $2 \mu \mathrm{g}$ of pcDNA3 empty/pCDNA3-Myc-tagged SPRED2 (gift from Dr. Yoshimura, Keio University, Tokyo) or Silencer select pre-designed siRNA SPRED2 (s47267) and control siRNA (4390844; Applied Biosystems). Transfection efficiency was quantified by PCR and/or western blot. In proliferation assays, PC3M cells were starved for $16 \mathrm{~h}$, then stimulated with FGF2 $\left(10 \mathrm{ng} \mathrm{ml}^{-1}\right)$ or $\mathrm{FM}$ and proliferation assessed by adding $10 \%$ WST-1 reagent (Roche Diagnostics). In migration assays, transfected cells prepared in basal medium were seeded in a BD migration chamber (Scientific Laboratory Supplies, Hessle, UK).
FM/FGF1 (10 $\left.\mathrm{ng} \mathrm{ml}^{-1}\right) / \mathrm{FGF} 2\left(10 \mathrm{ng} \mathrm{ml}^{-1}\right), \quad$ FGF8 $\left(10 \mathrm{ng} \mathrm{ml}^{-1}\right)$ and IGF(100 $\left.\mathrm{ng} \mathrm{ml}^{-1}\right)$ were used in the lower chamber for $24 \mathrm{~h}$. Migrating cells were fixed in methanol, stained with hematoxylin and counts averaged at $\times 20$ magnification over five different fields. Results for both assays are expressed as a fold change over un-induced controls and represent the mean of six experiments. Statistical analysis was performed by using two-tailed Student's $t$ test, $P<0.05$ being statistically significant.

Western blotting. Cells were lysed in Laemmli buffer and denatured. Samples were then separated using 10\% Bis-Tris precast gels (Invitrogen, Paisley, UK), followed by transfer to a PDVF membrane (GE Healthcare, Chalfont St Giles, UK). Antibody complexes were detected using HRP-conjugated secondary antibodies (Dako, Ely, UK). Protein bands were visualised using ECL (GE Healthcare). Primary antibodies used were pERK-E4 (sc7383), ERK-6G11 (sc-81458) and c-Myc-9E10 (sc-40) (Santa Cruz Biotechnologies, Heidelberg, Germany). $\alpha$-Tubulin (ab4074-100) was used as the loading control (Abcam, Cambridge, UK).

\section{RESULTS}

Expression of SPRED1 and SPRED2 in clinical prostate cancer. RNA was derived from microdissected tumours and quality tested by confirming expression of at least three housekeeping genes for each sample as previously described (Rogerson et al, 2008). Profiling for PCA 3 and EZH2 showed minimal expression in benign glands but high levels in grade 3,4 and 5 tumours consistent with the literature $(P<0.001$ for both) (van
A

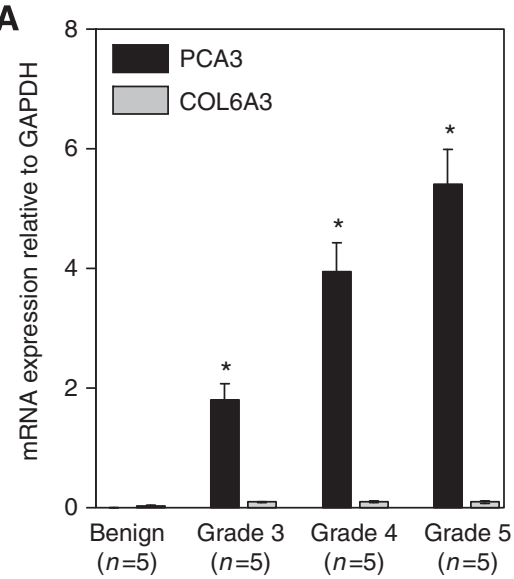

D

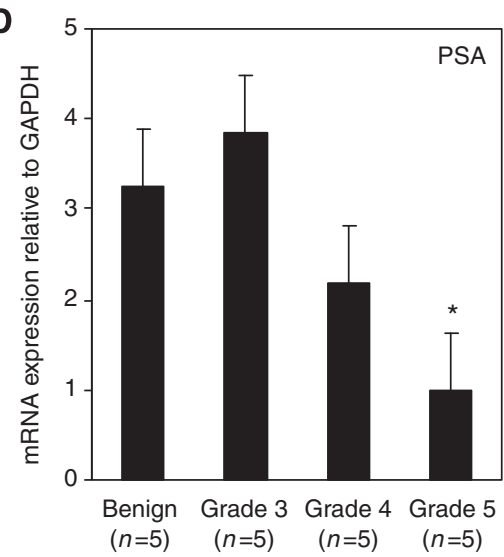

B

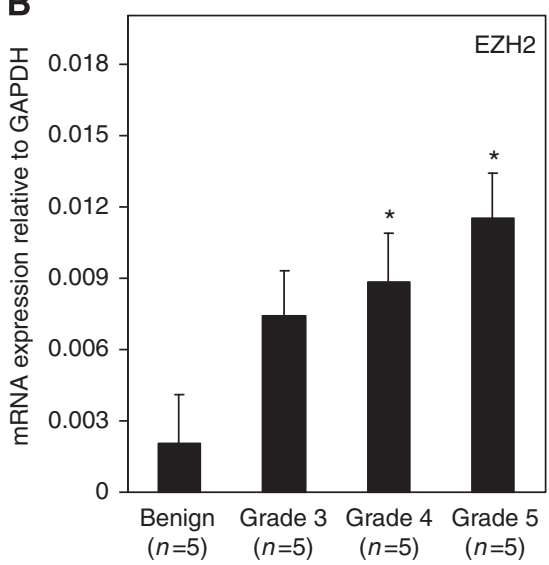

E

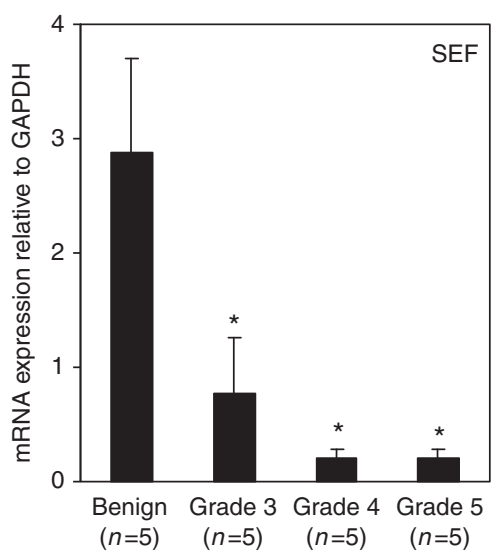

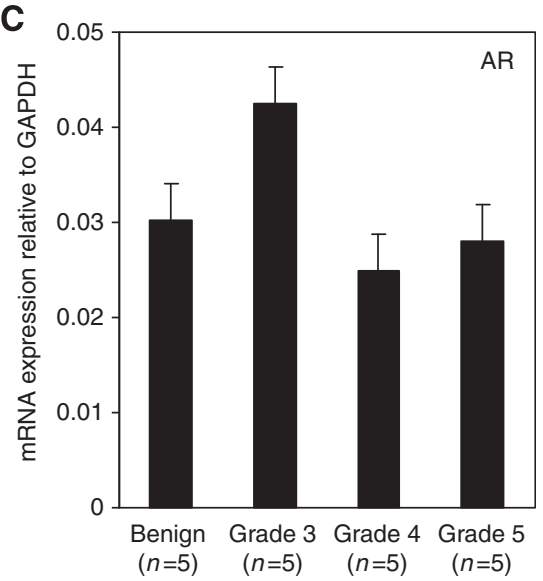

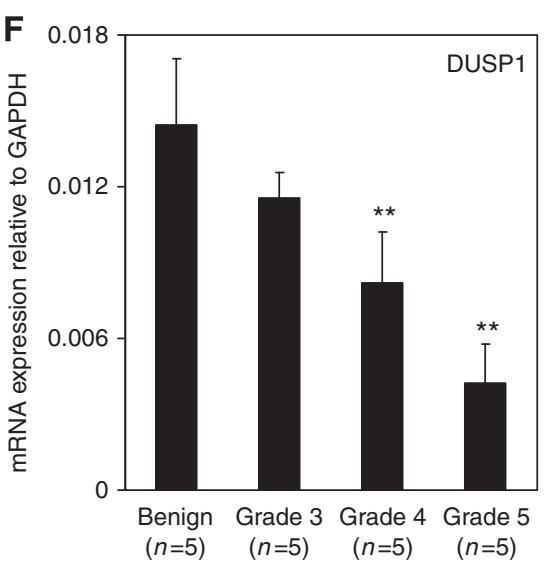

Figure 1. (A-F) Pooled transcript expression of PCA3, COL6A3, EZH2, AR, PSA, SEF and DUSP1 in microdissected benign glands and malignant tumours of different grades corrected to GAPDH. Results shown are the mean of three experiments repeated in triplicate ( ${ }^{\star} P<0.001,{ }^{\star \star} P<0.05$ ). 

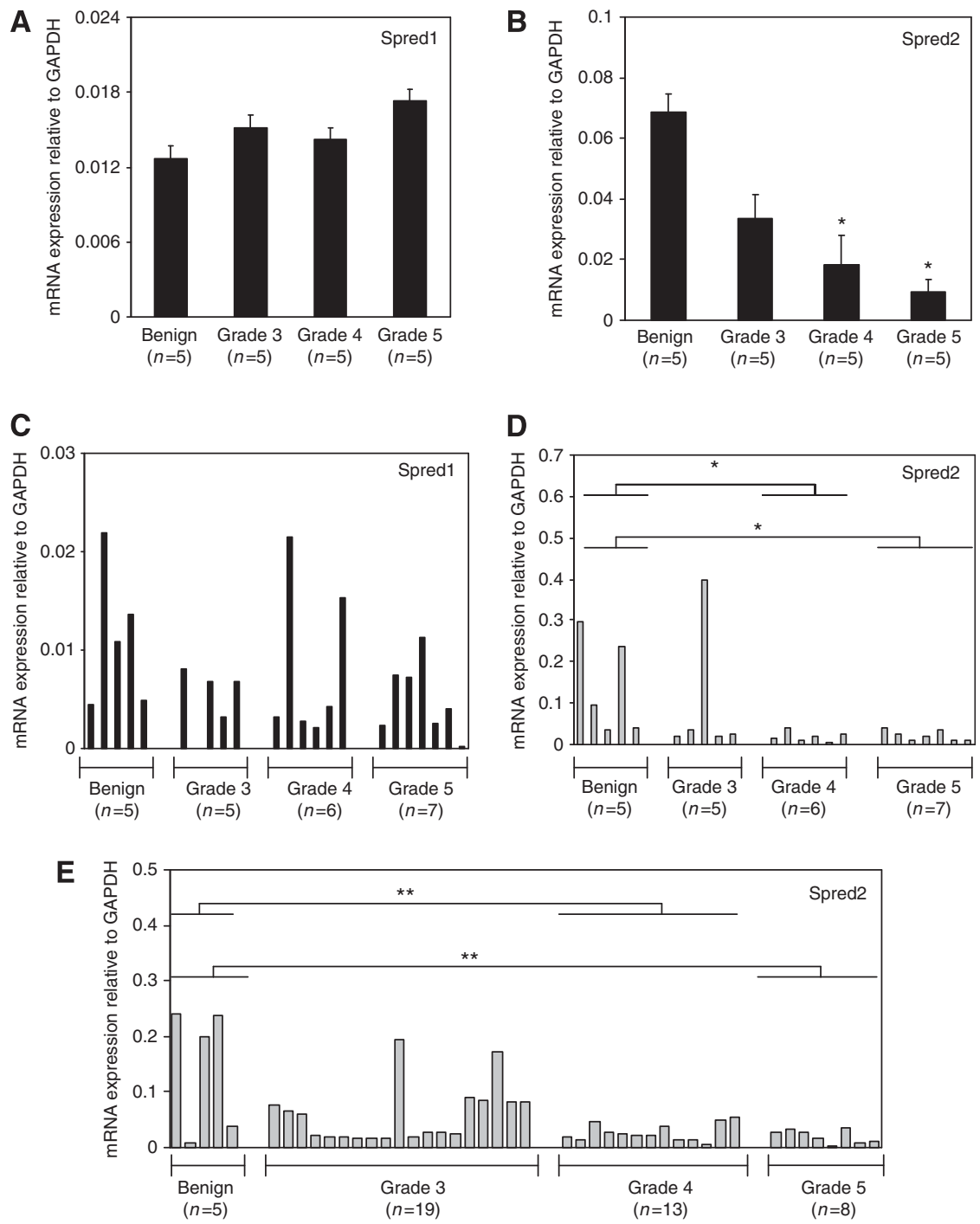

Figure 2. (A, B) Pooled transcript expression of SPRED1 and SPRED2 in microdissected benign glands and malignant tumours of different grades corrected to GAPDH. Results shown are the mean of three experiments repeated in triplicate. (C, D) Individual expression of benign and tumours in a second cohort of clinical samples tested for SPRED1 and SPRED2 transcript. One representative of three repeat experiments is shown. Each profile represents a tumour derived from an individual patient. (E) Expanded cohort of individual cases profiling SPRED2 expression. One representative of three repeat experiments is shown $\left({ }^{\star} P<0.05,{ }^{\star \star} P<0.01\right)$.

Leenders et al, 2007; Popa et al, 2007; Figures 1A and B). By contrast, the stromal marker COL6A3 was expressed at low levels in all the samples (Figure 1A). AR expression was not differentially expressed between tumours of different grades, consistent with previous reports (Ruizeveld et al, 1994; Figure 1C). By contrast, PSA was expressed at lower levels in high-grade tumours, again consistent with previous reports (Weir et al, 2000; Figure 1D). We next tested expression of the established negative regulators SEF and DUSP1. Here we observed high levels of SEF in benign glands but progressive downregulation in tumours of increasing Gleason grade $(P<0.01$; Figure $1 \mathrm{E})$. DUSP1 was similarly reduced in tumours compared with benign samples, particularly in Gleason grade 4 and 5 tumours $(P<0.05$; Figure $1 \mathrm{~F})$. These results are again consistent with previous studies in prostate cancer (Rauhala et al, 2005; Darby et al, 2009). SPRED1 and 2 have not previously been investigated in prostate cancer. We found no appreciable difference in SPRED1 transcript comparing benign with tumour samples or indeed between different tumour grades (Figure 2A). By contrast, we observed high levels of SPRED2 mRNA in benign glands but lower levels of expression in tumours $(P<0.05)$ (Figure $2 \mathrm{~B})$. This difference was particularly apparent in higher grade tumours. We then investigated SPRED expression in a separate set of microdissected tumours ( $n=5$ benign, $n=5$ Gleason 3, $n=6$ Gleason 4 and $n=7$ Gleason 5). Here we again found similar SPRED1 expression in benign and tumour glands (Figure 2C). By contrast, SPRED2 was expressed at high or moderate levels in the majority of benign glands. Among tumours, however, the majority had reduced SPRED2 expression, and this was particularly apparent in the grade 4 and 5 tumours $(P=0.03$ and $P=0.02$, respectively), consistent with the findings from our initial tumour set (Figure 2D). To further validate the observed downregulation of SPRED2, we re-tested expression in an expanded cohort of cases (additional $n=5$ benign, $n=19$ Gleason 3, $n=13$ Gleason 4 and $n=8$ Gleason 5 ; Figure 2E). Here again we found a significant downregulation of SPRED2, particularly in grade 4 and grade 5 tumours compared with benign glands $(P=0.001$ and $P=0.008$, respectively).

Functional effect of manipulating SPRED2 in prostate cancer. The functional role of SPRED2 has not previously been investigated in prostate cancer. We first tested whether SPRED2 was androgen regulated. AR-positive LNCaP cells were treated 
with synthetic androgens and SPRED2 and PSA (control) levels assayed by real-time PCR. In this experiment, PSA transcript was induced by nearly 10 -fold $(P<0.001)$, while SPRED2 mRNA levels remained unchanged (Figure $3 \mathrm{~A})$. The effect of manipulating SPRED2 was next tested using an overexpression plasmid in PC3M prostate cells (Figure 3B). Transient transfection with SPRED2 effectively blocked pERK stimulation following stimulation with FM (Figure 3B). By contrast, pERK levels were rapidly induced in empty vector-transfected cells. In WST-1 assays, stimulation of empty vector PC3M-transfected cells with FGF2 or FM resulted in a four-fold increase in proliferation over 48 hours compared with basal levels of proliferation at time 0 . Cells transfected with SPRED2 however failed to respond to FGF2 or FM stimulation
(Figures $3 \mathrm{C}$ and D). In migration assays, compared with controls, SPRED2 overexpression effectively blocked stimulation by different FGFs, IGF and full media (Figure 3E). We next tested the converse effect of suppressing SPRED2 using siRNA knockdown in PC3M cells. We have been unable to identify a suitable antibody to detect endogenous SPRED2 protein in our cell line. We therefore confirmed effective suppression of SPRED2 but not SPRED1 by siRNA using PCR (Figure 3F). In these experiments, SPRED2 silencing enhanced the response to different ligands compared with scramble-transfected controls (Figure 3G). These results suggest that SPRED2 is a putative negative regulator in prostate cancer cells and is capable of attenuating MAPK stimulation by diverse growth factor ligands.
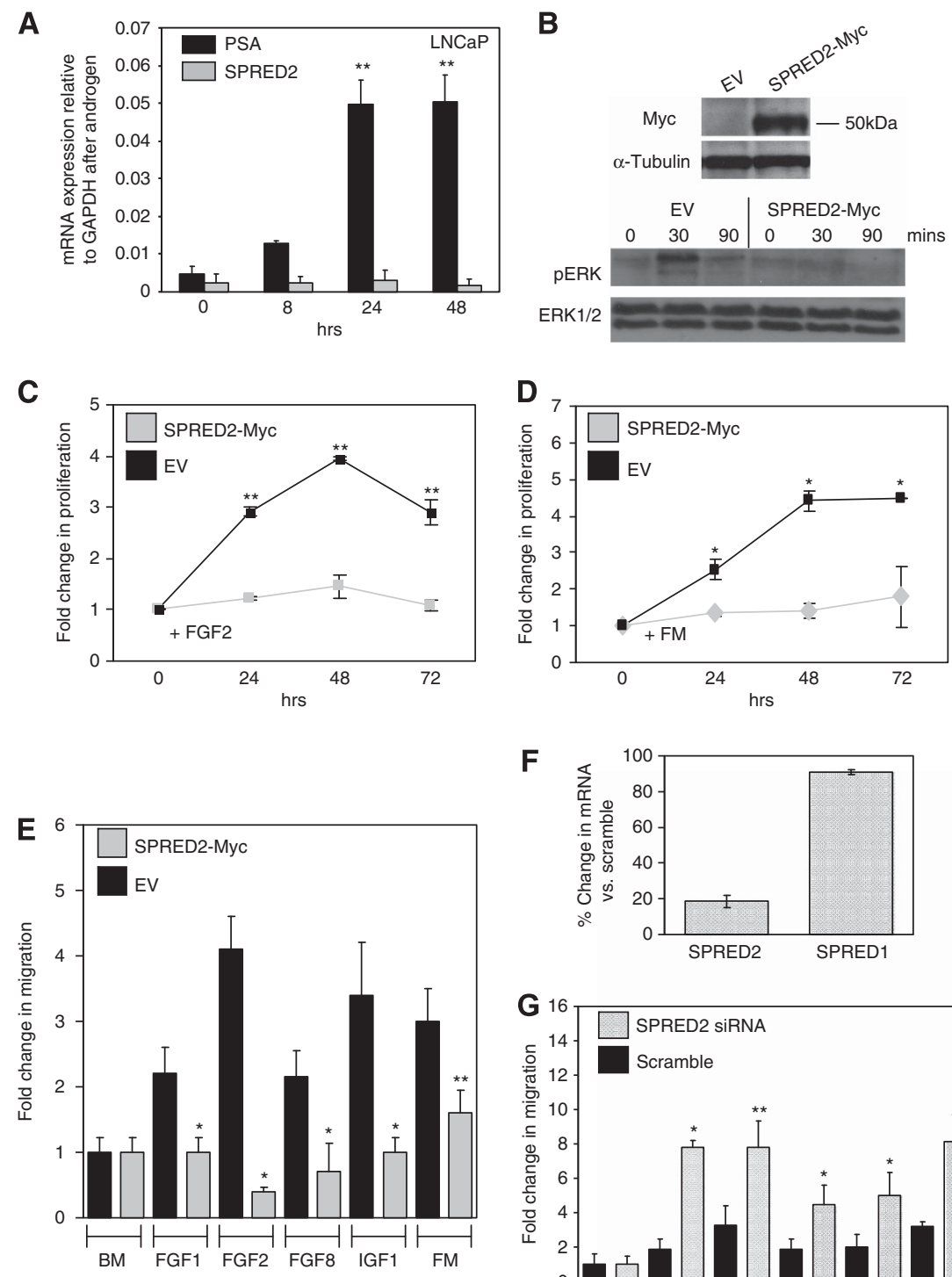

Figure 3. (A) Relative expression of SPRED2 and PSA mRNA after treatment with synthetic androgen R1881 in LNCaP prostate cells. Results show the mean of three experiments repeated in triplicate. (B) Western blot confirming expression of the SPRED2-Myc construct in PC3M cells. The blot was then stripped and re-probed for $\alpha$-tubulin. Western blot for PERK and ERK1/2 with transfection of SPRED2-Myc or empty vector plasmid (EV). One of three experiments is shown. (C,D) Proliferation assay in PC3M cells transfected with SPRED2-Myc or EV and stimulated with either FGF2 or full media (FM). Results shown are fold changes over basal proliferation levels and represent the mean of three experiments. (E) Migration assay in PC3M cells transfected with SPRED2-Myc or EV plasmid and stimulated with FGF1/2/8, IGF and FM. Results shown are fold changes over basal levels and represent the mean of three experiments. (F) Specific knock down of SPRED2 mRNA by targeted siRNA with intact SPRED1 expression in PC3M cells. Expression shown is percentage change in mRNA compared with scramble (SCR)-transfected controls. (G) Migration assay in PC3M cells transfected with SPRED2 siRNA or SCR and stimulated with FGF1/2/8, IGF and FM. Results shown are fold changes over basal levels and represent the mean of three experiments $\left({ }^{\star} P<0.001,{ }^{\star \star} P<0.05\right)$. 
Interpretation. Only one study (in hepatocellular cancer) has reported on changes in SPRED expression in a human malignancy. In that study, both SPREDs were downregulated in cancer (Yoshida et al, 2006). In the present study, we unexpectedly observed loss of SPRED2 but not SPRED1 in prostate cancers compared with benign glands. Interrogation of a publically available transcript data set (MSKCC Prostate Oncogenome Project) also appears to corroborate this apparent dichotomy (Taylor et al, 2010). Tumours in this data set were not isolated by laser microdissection or by distinct grades. Nevertheless, in comparison to normal controls, SPRED2 was downregulated in $28 \%$ of primary tumours and $47 \%$ of metastatic tumours, while SPRED1 expression was unchanged. We have not been able to reliably test protein expression of SPREDs in prostate tissue. Different SPRED2 antibodies were extensively tested by western blot and immunohistochemistry, but none showed sufficient sensitivity and specificity. Development of a suitable antibody is a key aim of our current laboratory work.

Ma et al (2011) recently demonstrated the phenotypic effect of manipulating SPRED2 in human hepatocellular cancer cells whereby increased expression inhibited cell proliferation and migration, while suppression enhanced in vivo tumour growth. In the present study, we were able to recapitulate these effects in prostate cancer. SPRED2 overexpression blocked ERK phosphorylation and inhibited diverse growth factor-induced proliferation and migration. By contrast, suppression of SPRED2 enhanced growth factor-mediated mitogenic effects, similar to the previous findings in hepatocellular cancer.

In summary we present first evidence of specific SPRED2 downregulation in prostate cancer and a role in inhibiting mitogenic MAPK signalling. We are currently validating these findings in larger cohorts and investigating SPRED2 as a prognostic and/or therapeutic marker. The current results, however, provide a convincing rationale for further studies to investigate SPRED2 as a candidate tumour suppressor in prostate cancer.

\section{ACKNOWLEDGEMENTS}

This study was funded by Cancer Research UK (C1435/A4114), the Evelyn trust and The Ralph Shackman Trust. NK is funded by The Urology Foundation Research Scholarship, a RCS (England) Research Fellowship and a Raymond and Beverley Sackler Studentship (University of Cambridge). We are grateful to $\mathrm{Mr} \mathrm{A}$ Joseph for providing technical assistance.

\section{REFERENCES}

Brognard J, Hunter T (2011) Protein kinase signaling networks in cancer. Curr Opin Genet Dev 21: 4-11.

Bundschu K, Walter U, Schuh K (2007) Getting a first clue about SPRED functions. Bioessays 29: 897-907.
Darby S, Murphy T, Thomas H, Robson CN, Leung HY, Mathers ME, Gnanapragasam VJ (2009) Similar expression to FGF (Sef) inhibits fibroblast growth factor-induced tumourigenic behaviour in prostate cancer cells and is down-regulated in aggressive clinical disease. $\mathrm{Br} J$ Cancer 101: 1891-1899.

Ma XN, Liu XY, Yang YF, Xiao FJ, Li QF, Yan J, Zhang QW, Wang LS, Li XY, Wang H (2011) Regulation of human hepatocellular carcinoma cells by Spred2 and correlative studies on its mechanism. Biochem Biophys Res Commun 410: 803-808.

Murphy T, Hori S, Sewell J, Gnanapragasam VJ (2010) Expression and functional role of negative signalling regulators in tumour development and progression. Int J Cancer 127: 2491-2499.

Popa I, Fradet Y, Beaudry G, Hovington H, Beaudry G, Têtu B (2007) Identification of PCA3 (DD3) in prostatic carcinoma by in situ hybridization. Mod Pathol 20: 1121-1127.

Rauhala HE, Porkka KP, Tolonen TT, Martikainen PM, Tammela TL, Visakorpi T (2005) Dual-specificity phosphatase 1 and serum/ glucocorticoid-regulated kinase are downregulated in prostate cancer. Int J Cancer 117: 738-745.

Rogerson L, Darby S, Jabbar T, Mathers ME, Leung HY, Robson CN, Sahadevan K, O’Toole K, Gnanapragasam VJ (2008) Application of transcript profiling in formalin-fixed paraffin-embedded diagnostic prostate cancer needle biopsies. BJU Int 102: 364-370.

Ruizeveld de Winter JA, Janssen PJ, Sleddens HM, Verleun-Mooijman MC, Trapman J, Brinkmann AO, Santerse AB, Schröder FH, van der Kwast TH (1994) Androgen receptor status in localized and locally progressive hormone refractory human prostate cancer. Am J Pathol 144: 735-746.

Taylor BS, Schultz N, Hieronymus H, Gopalan A, Xiao Y, Carver BS, Arora VK, Kaushik P, Cerami E, Reva B, Antipin Y, Mitsiades N, Landers T, Dolgalev I, Major JE, Wilson M, Socci ND, Lash AE, Heguy A, Eastham JA, Scher HI, Reuter VE, Scardino PT, Sander C, Sawyers CL, Gerald WL (2010) Integrative genomic profiling of human prostate cancer. Cancer Cell 18: 11-22.

Valencia T, Joseph A, Kachroo N, Darby S, Meakin S, Gnanapragasam VJ (2011) Role and expression of FRS2 and FRS3 in prostate cancer. BMC Cancer 11: 484.

van Leenders GJ, Dukers D, Hessels D, van den Kieboom SW, Hulsbergen CA, Witjes JA, Otte AP, Meijer CJ, Raaphorst FM (2007) Polycomb-group oncogenes EZH2, BMI1, and RING1 are overexpressed in prostate cancer with adverse pathologic and clinical features. Eur Urol 52: 455-463.

Wakioka T, Sasaki A, Kato R, Shouda T, Matsumoto A, Miyoshi K, Tsuneoka M, Komiya S, Baron R, Yoshimura A (2001) Spred is a Sprouty-related suppressor of Ras signalling. Nature 412: 647-651.

Weir EG, Partin AW, Epstein JI (2000) Correlation of serum prostate specific antigen and quantitative immunohistochemistry. J Urol 163: 1739-1742.

Yoshida T, Hisamoto T, Akiba J, Koga H, Nakamura K, Tokunaga Y, Hanada S, Kumemura H, Maeyama M, Harada M, Ogata H, Yano H, Kojiro M, Ueno T, Yoshimura A, Sata M (2006) Spreds inhibitors of the Ras/ERK signal transduction, are dysregulated in human hepatocellular carcinoma and linked to the malignant phenotype of tumors. Oncogene 25: 6056-6066.

This work is published under the standard license to publish agreement. After 12 months the work will become freely available and the license terms will switch to a Creative Commons AttributionNonCommercial-Share Alike 3.0 Unported License. 